\title{
The Nonlinear Distortions in the Oscillatory System of Generator on CFOA
}

\author{
Yuriy Konstantinovich Rybin \\ National Research Tomsk Polytechnic University, Tomsk 634050, Russia \\ Correspondence should be addressed to Yuriy Konstantinovich Rybin, rybin@tpu.ru \\ Received 28 January 2012; Revised 18 March 2012; Accepted 23 March 2012 \\ Academic Editor: Abhirup Lahiri
}

Copyright (C 2012 Yuriy Konstantinovich Rybin. This is an open access article distributed under the Creative Commons Attribution License, which permits unrestricted use, distribution, and reproduction in any medium, provided the original work is properly cited.

\begin{abstract}
In recent years, many articles came out where one could find the analysis of oscillatory systems of electric sinusoid signals generators with amplifiers called CFOA—current feedback operational amplifiers. As a rule, the analysis of such systems is made by applying mathematical modeling methods on the basis of the amplifier linear model, which does not allow estimating advantages and disadvantages of the systems realized with those amplifiers in comparison with classical systems. A nonlinear model of a current feedback operational amplifier (CFOA) is introduced in the paper; nonlinearity of "current mirror" is reflected in the form of current double limiting. The analysis of two known oscillatory systems has been carried out with the use of this nonlinear model. Dependence between current limiting level, output voltage amplitude, and maximum oscillation frequency has been obtained. The paper shows that output current limiting under current output connection of capacitive load reduces frequency range and output voltage amplitude considerably and increases harmonic distortions in comparison with classical oscillatory systems. The research done has found that the application of new amplifiers does not give considerable advantages to the oscillatory systems with CFOA.
\end{abstract}

\section{Introduction}

In the end of the last century a new operational amplifier in the monolithic form appeared in the market of electronic components. Its distinctive features, in comparison with traditional operational amplifiers, were inverting input with low input resistance, additional output with high output resistance, frequency range extension, and very fast large signal response. The amplifier was called a current feedback operational amplifier (CFOA). Typically, these amplifiers perform on the complementary bipolar technology on heterogeneous symmetric $\mathrm{p}-\mathrm{n}-\mathrm{p}$ and $\mathrm{n}-\mathrm{p}-\mathrm{n}$ transistors.

Many companies have developed the manufacture of the amplifiers. The examples of the amplifiers are AD844, OPA622, and others. The description of the circuit technology of the new amplifiers is given in the materials of manufacturing companies $[1,2]$. There is a detailed description of AD844 amplifier (Rybin [3]). Naturally, such amplifiers provoked great interest amidst developers of electronic devices, for example, oscillatory systems (OSs) of electric signals generators. Many works came out where different variants of OS of sinusoid oscillations were given (Abuelma' atti $[4,5]$, Abuelma' atti and Al-Shahrani [6], Soliman [7, 8], and Martínez et al. [9].) There one could find the analysis of conditions of oscillation excitation and establishing (Abuelma' atti [4]), their advantages and disadvantages. However, the analysis was done on the basis of linear models of CFOA, so it has limited use and does not allow disclosing all advantages and disadvantages.

Taking into account the fact that OS is a basis of any electric signals generator, as periodic oscillation is excited and established there, and OS determines their form and basic parameters, the author makes an attempt to analyze OS on the basis of CFOA nonlinear model exemplified by two OSs.

\section{Current Feedback Operational Amplifier}

Figure 1 shows a non-linear equivalent circuit CFOA amplifier of $A D 844$ type. The circuit consists of two voltage followers $D A 1$ and $D A 2$, current-controlled current supply 


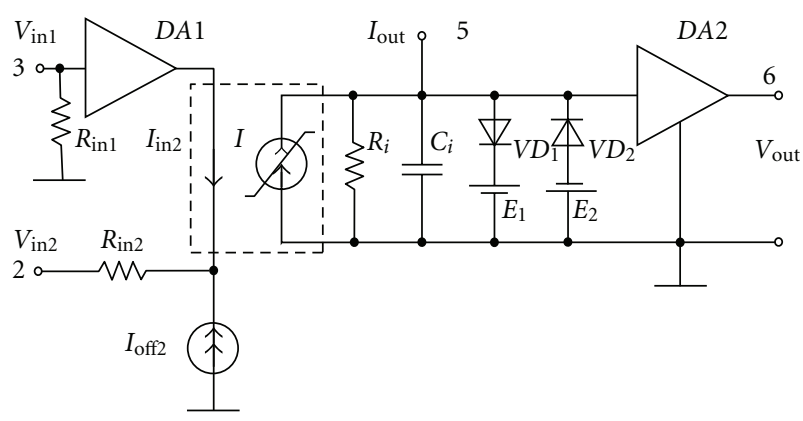

FIgURE 1: Nonlinear equivalent circuit CFOA of AD844 type.

done according to the "current mirror" (CM) circuit, RCcircuit imitating inertia, and double output voltage limiter. One amplifier input $\left(V_{\text {in } 1}\right)$ is noninverting with high input resistance (potential input); the other $\left(V_{\text {in2 }}\right)$ is currentinverting with low input resistance $\left(R_{\mathrm{in} 2}\right)$. To represent different input resistances the circuit contains voltage follower $D A 1$. It is known that the special feature of this amplifier is its two outputs: "current" output $\left(I_{\text {out }}\right)$ and "potential" output $\left(U_{\text {out }}\right)$. A usual potential output $\left(V_{\text {out }}\right)$ is made at the follower $D A 2$ output after the output voltage limiter on diodes VD1 and VD2 with voltage sources E1 and E2. Current output $\left(I_{\text {out }}\right)$ has high output resistance $R i$. It is important that the circuit has current limiter shown in CM (current mirror) block. Output current $\left(I_{\text {out }}\right)$ is equal to input current at inverting input due to the use of "current mirror" in the circuit, but it is limited by maximum current $I=\left\{I_{1}\right.$ if $\left|I_{1}\right| \leq I_{\max } ; I_{\max }$ if $I_{1}>I_{\max } ;-I_{\max }$ if $\left.I_{1}<-I_{\max }\right\}$. That makes the circuit different from other known models of CFOA (Thomas H. Lee).

Limitation of current and voltage turns the equivalent circuit into a nonlinear one with piecewise-linear elements. At the same time in the amplifier areas of linear amplification and restriction are formed. This allows analyzing the conditions of excitation of oscillations in the oscillator. When the excitation processes take place in the linear area of characteristic, restriction is used to determine the maximum amplitude and its dependence on frequency of oscillations.

Figure 2 gives a representation of the amplifier with potential (3) and current (2) inputs and potential (6) and current (5) outputs.

To build OS one can use the new amplifier practically in all known OSs replacing VFOA_voltage feedback operational amplifier by CFOA. The circuit analysis can be done by known methods. Interesting are those OSs that can be realized only with CFOA and cannot be repeated with usual amplifiers.

\section{Analysis of Oscillatory Systems with CFOA}

Figure 3 shows the first example of OS generator circuit with one amplifier. Specifically, Figure 3(a) shows a circuit of a known OS with Wien-Robinson bridge done with an amplifier with potential feedback VFOA; Figure 3(b) shows new OS $[4,9]$. One can see that the new circuit uses "current

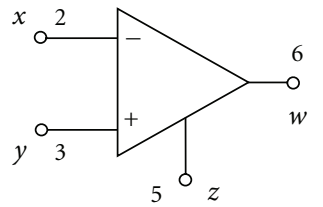

Figure 2: Representations of CFOA of AD844 type at electric circuits with numeric and corresponding letter symbols of outputs.

output" connected with output 5 of the microcircuit. It can also be observed that the circuits contain different numbers of passive elements. The classical circuit has 6 of them, and the new one has only 4. Certainly, the difference of two resistors is not essential. The point is different. To change oscillation frequency one usually uses two or even four adjustable elements (two resistors and/or two capacitors), for example, doubled continuously adjustable capacitor or doubled potentiometer. One or both capacitors should be isolated from the common wire. But continuously adjustable capacitors with mechanically adjusted capacitance are bulky elements with high stray capacitance per device case, which influences significantly generation conditions and oscillation frequencies, especially in low capacitances. This drawback applies equally to mechanic potentiometers also used to vary frequencies. In the new circuit both capacitors/potentiometers can be connected with each other by one of the outputs with common wire, and that could simplify their manufacture and application considerably.

Let us analyze OS shown in Figure 3(b) to estimate conditions of sinusoid oscillation generation. With this aim in view we put down the OS equation set with ideal CFOA: $V_{z}=I_{z} Z_{2}, V_{x}=I_{x} Z_{1}, V_{x}=V_{y}=V_{w}, I_{x}=I_{z}$ where $Z_{1}(s)=R_{1}+1 / s C_{1}, Z_{2}(s)=R_{2} /\left(1+s R_{2} C_{2}\right), s=\sigma+j \omega$.

Substituting currents $I_{z}$ and $I_{x}$ from the first and second equations into the fourth with a glance at the third one, we obtain $V_{w}\left[Z_{1}(s)-Z_{2}(s)\right]=0$. After substituting circuit resistances we put down OS characteristic equation

$$
s^{2} R_{1} C_{1} R_{2} C_{2}+s\left(R_{2} C_{2}+R_{1} C_{1}-R_{2} C_{1}\right)+1=0 .
$$

Analyzing the obtained equation, we find conditions of phase and amplitude balance (the Barkhausen criterion) realization:

$$
\begin{gathered}
\omega=\frac{1}{\sqrt{R_{1} C_{1} R_{2} C_{2}}}, \\
R_{2} C_{2}+R_{1} C_{1}-R_{2} C_{1}=0 .
\end{gathered}
$$

The second condition, amplitude balance, does not differ from the condition obtained in [4]. So it is satisfied under $R_{2}=2 R_{1}$ and $C_{2}=0.5 C_{1}$. As one can see, both oscillation frequency and amplitude balance depend on the same elements: $R_{1}, C_{1}, R_{2}$, and $C_{2}$. Therefore, there is a connection between oscillation frequency and amplitude. In this case oscillation would not be isochronous, as elements parameters instability would be accompanied by simultaneous changing of oscillation amplitude and frequency.

One can eliminate the dependence of frequency on the oscillation amplitude and at the same time apply frequency 


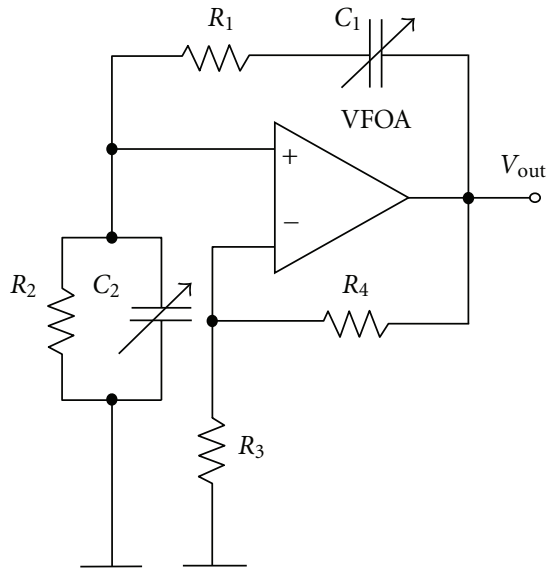

(a)

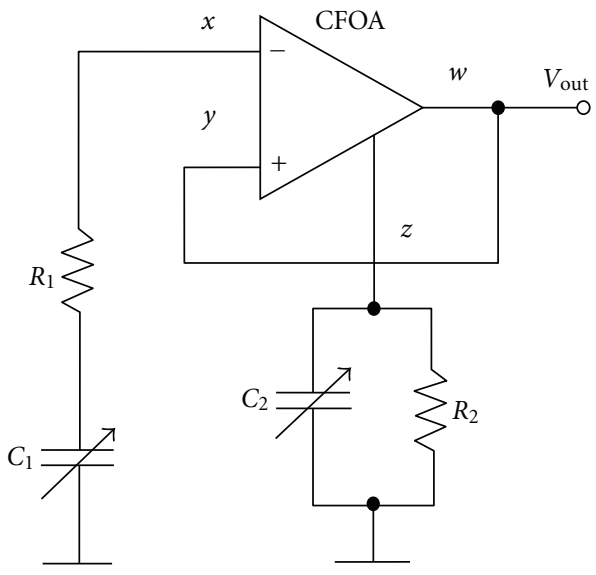

(b)

FIGURE 3: Oscillation systems circuits: (a) with usual amplifier VFOA; (b) with CFOA.

driving elements with equal parameters in enhanced OS shown in Figure 4.

After similar transformations as for the circuit in Figure 3(b) we get the OS characteristic equation

$$
s^{2} R_{1} C_{1} R_{2} C_{2}+s\left[R_{2} C_{2}+R_{1} C_{1}-\beta R_{1} C_{2}\right]+1=0 .
$$

Whence we find conditions of amplitude and phase balances:

$$
\begin{gathered}
\omega=\frac{1}{\sqrt{R_{1} C_{1} R_{1} C_{2}}}, \\
R_{2} C_{2}+R_{1} C_{1}-\beta R_{1} C_{2}=0 .
\end{gathered}
$$

In this circuit the conditions for potential oscillation generation are satisfied under $R_{1}=R_{2}=R, C_{1}=C_{2}=C$, and $\beta=2$. Then, oscillation frequency is equal to $\omega=$ $1 / R C$. It is obvious that circuit $\beta$ should be an active circuit amplifier with an amplification factor of two. OS with such parameters is shown in Figure 4(b). It is formed by elements with equal parameters of frequency driving elements, which is its advantage. Its amplitude balance, regardless of phase balance, is provided by resistance of additional resistors $R_{3}$ and $R_{4}$, between which correlation $R_{4}=2 R_{3}$ should be satisfied in stationary mode. The advantage of this circuit in comparison with OS circuit in Figure 3(b) is in the fact that its oscillation is isochronous as being controlled by oscillation amplitude does not change its frequency.

Interesting is OS of two integrators, each of which is realized with CFOA [4]. Figure 5 exemplifies an OS with two integrators: classical and new circuits. To compare the circuits correctly, both of them are realized with CFOA. The conditions of amplitude and phase balances for the first circuit are known. It is not difficult to put the conditions down for the second circuit as well on the basis of transfer function of two frequency-dependent active elements realized with amplifiers $D A 1$ and $D A 2$ :

$$
\begin{aligned}
K_{1}(s) & =\frac{Z_{2}(s)}{Z_{2}(s)-R_{1}}=\frac{R_{2} /\left(1+s R_{2} C_{1}\right)}{R_{2} /\left(1+s R_{2} C_{1}\right)-R_{1}} \\
& =\frac{R_{2}}{R_{2}-R_{1}+s R_{2} R_{1} C_{1}}, \\
K_{2}(s) & =\frac{Z_{C 2}(s)}{R_{3}}=\frac{1}{s R_{3} C_{2}} .
\end{aligned}
$$

Thus, in this circuit active linear frequency-dependent elements, the first (under $R_{2}=R_{1}=R$ ) and the second, are integrators. OS characteristic equation and equations for amplitude and phase balances take the following form

$$
\begin{gathered}
Q(s)=s^{2} R_{1} R_{2} R_{3} C_{1} C_{2}+s\left[R_{1}-R_{2}\right] R_{3} C_{2}+R_{2}, \\
\omega=\sqrt{\frac{R_{2}}{R_{1} R_{2} R_{3} C_{1} C_{2}}}=\frac{1}{\sqrt{R_{1} R_{3} C_{1} C_{2}}}, \\
R_{1}-R_{2}=0 .
\end{gathered}
$$

Amplitude balance in the system is reached under equality of resistances $R_{1}$ and $R_{2}$, while phase balance and, consequently, frequency in this circuit does not depend on resistance $R_{2}$. That allows controlling oscillation amplitude without changing frequency by changing resistance $R_{2}$. At the same time, changing resistance $R_{3}$, one can control oscillation frequency without changing amplitude, though changing frequency would depend on resistor's resistance under complex law. Thus, the circuit gives the possibility to control the conditions of oscillation excitation and frequency changing with the help of resistors connected by the common wire.

Let us compare OS for the realization of their potential resources.

Essentially these schemes use different types of feedback. In the circuit shown in Figure 5(a) in each amplifier external voltage negative feedback is applied from the output of $w$ to 


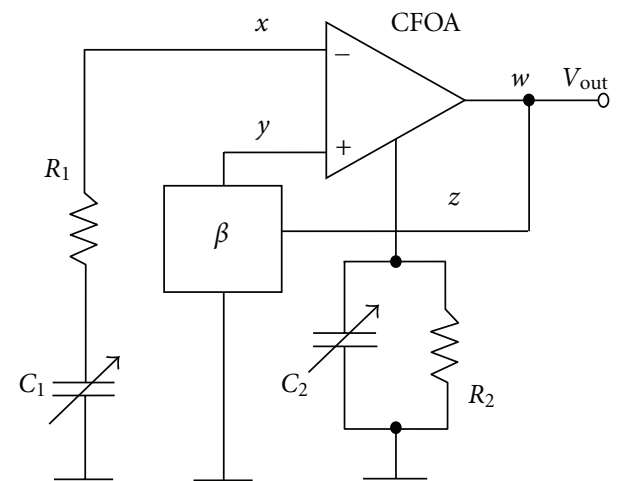

(a)

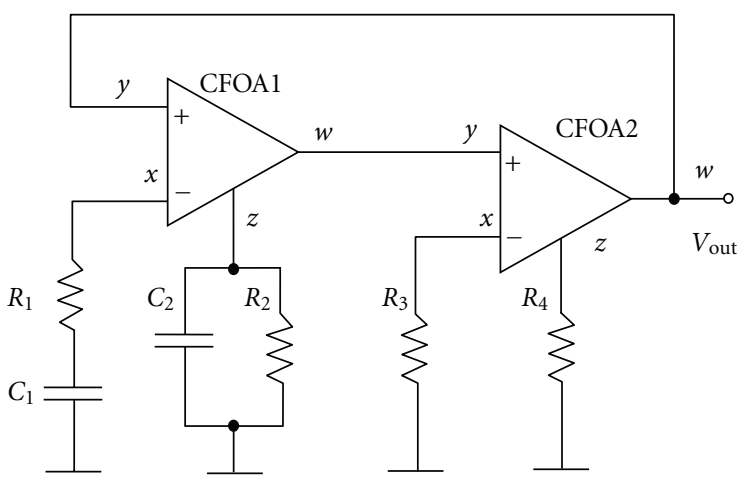

(b)

FIGURE 4: Enhanced oscillation systems with CFOA: (a) simplified, with feedback circuit $\beta$; (b) fundamental, with two CFOA, one of which is used as circuit $\beta$.

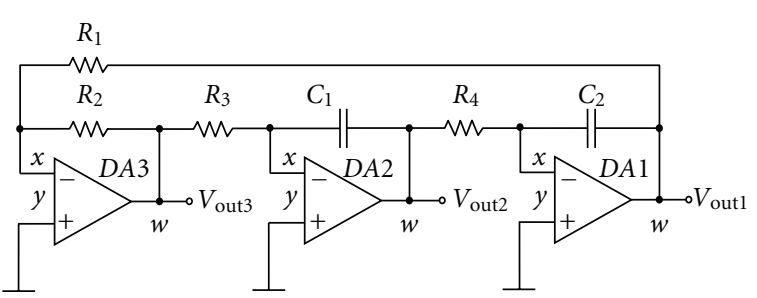

(a)

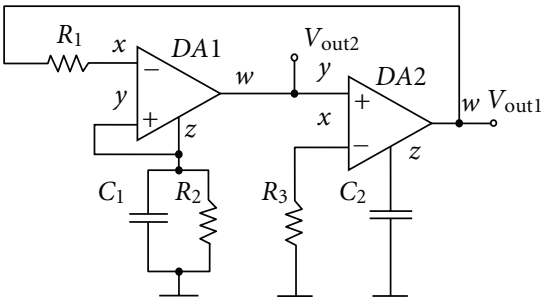

(b)

Figure 5: Oscillation systems of two integrators with CFOA: (a) classical and (b) new circuits.

an input $x$, and in Figure 5(b) current feedback on input $x$. It is known that the input $x$ in the CFOA amplifiers is connected inside the circuit with $\mathrm{p}-\mathrm{n}-\mathrm{p}$ and $\mathrm{n}-\mathrm{p}-\mathrm{n}$ emitters of the input transistors. In the latter case, on any resistance connected to the emitters the current feedback appears.

Circuit $5 b$ has less active and passive elements. It needs only two amplifiers and five passive elements, but it is important to take into consideration that today the difference of one amplifier in the monolithic form and two-three passive components when making industrial generator is not essential. Moreover, in circuit $5 \mathrm{a}$ the additional amplifier makes it possible to get one more output.

More important is OS comparison for non-linear distortions level. Low non-linear distortions level approximates output voltage to sinusoid form. It is output voltage sinusoid form that becomes the objective of making sinusoid oscillation generators. Non-linear distortions reduction in the first circuit is reached by several factors:

(i) introducing external voltage feedback from the output $w$ to the input $x$

(ii) Absence of in-phase distortions, as amplifiers inputs work under quite low signals in contrast to the new circuit where both amplifiers inputs are under common-mode voltage equal to output voltage, which may cause distortions; It is known that amplifiers of CFOA type have relatively low common mode rejection ratio; (iii) reduction of influence of distortions of the input and output voltage follower, as they are covered by local and common negative feedback. The point is that the followers are output stage in the structure of usual amplifiers with potential feedback, so in the first-classical circuit their distortions influence on output voltage distortions is effectively reduced by the external feedback; in the new circuit the voltage followers in the structure of CFOA are in the circuit of positive feedback and their distortions are not suppressed.

To back this statement an experiment was carried out where higher harmonic rates of the main blocks of generators-output integrators were measured. For the experiment amplifiers of $A D 844$ type under supply voltage $\pm 10 \mathrm{~V}$, resistors with resistance $20 \mathrm{k} \Omega$ and capacitors with capacitance $32.85 \mathrm{nF}$ were chosen as amplifiers. Measurements were taken under frequency $200 \mathrm{~Hz}$ and output voltage amplitude $5 \mathrm{~V}$. Generator GS-50 (THD $<-120 \mathrm{~dB})$ and rejection filter with rejection frequency $200 \mathrm{~Hz}$ were used in the experiment. The results of the experiment are given in Table 1.

As one can see from Table 1, the classical integrator has the evident advantage of harmonic rate level. Its distortions are $14 \cdots 30 \mathrm{~dB}$ less.

Another important advantage of the OS with CFOA mentioned in the materials of the company manufacturer is its better frequency qualities. These amplifiers, when using 
TABLE 1: Comparison of integrators for higher harmonic level, dB.

\begin{tabular}{lcc}
\hline Harmonic rate & $\begin{array}{r}\text { Integrator of } D A 1, R_{4}, \\
C_{2} \text {, Figure } 5(\mathrm{a})\end{array}$ & $\begin{array}{c}\text { Integrator of } D A 2, R_{3}, \\
C_{2}, \text { for Figure } 5(\mathrm{~b})\end{array}$ \\
\hline$K_{2 r}$ & -85.3 & -68.9 \\
$K_{3 r}$ & -90.4 & -57.8 \\
$K_{4 r}$ & -94.2 & -80.5 \\
\hline
\end{tabular}

potential output (output 6 in Figure 1), have wide range of amplified frequencies (up to $60 \mathrm{MHz}$ ). Let us see whether we could realize this advantage of OS using current output.

To apply CFOA with current output (output 5) it is important to know its output load capacity. The manufacturer of the amplifier [1] certifies maximum input current for inverting input $x$ not more than $5 \mathrm{~mA}$. The same would be maximum current for output $z$. Experimental measuring of maximum output current for output $z$ when signal limit for current comes gives the following values: $I_{\max }=2.5 \mathrm{~mA}$ under supply voltage $\pm 10 \mathrm{~V}$ and $I_{\max }=3.5 \mathrm{~mA}$ under $\pm 15 \mathrm{~V}$. When connecting to the current output of outer elements current in them is limited exactly by these maximum values. Limit for current leads to limit for potential amplitude of integrator output voltage under given frequency. Let us calculate the dependence of integrator output voltage amplitude on oscillation frequency. The relation between these values with a glance at oscillation sinusoid form is given by the known equation $I_{\max }=2 \pi f C U_{m}$. This formula connects maximum current value, integrator output voltage amplitude, frequency, and capacitance of capacitor.

The minimum value of the capacitance of the integrator cannot be less than the capacitance of the output $z$ (transcapacitance), that is, it cannot be less than $4.5 \mathrm{pF}$ for the amplifier AD844. This capacity is always present and will determine the error of output oscillation frequency of the generator.

Let us use the formula to estimate whether we could realize the amplifier potential frequency properties in integrator circuit. According to the manufacturer [1] data one can get the voltage of $20 \mathrm{~V}$ peak-peak at the potential output under frequency $20 \mathrm{MHz}$. With the sinusoid form this voltage corresponds to double output voltage amplitude, that is, equal to $10 \mathrm{~V}$. Let us put these values to the last formula and estimate potential value of the integrator capacitor capacitance under this frequency and voltage amplitude with the formula $C=I_{\max } / 2 \pi f U_{m}$. The value of the integrator capacitor capacitance is equal to $5.6 \mathrm{pF}$. The value is too small for frequency driving capacitance and can be compared with output stray capacitance of current output $(4.5 \mathrm{pF})$, so it cannot be regarded as the integrator capacitance.

Real potential values should be chosen with a glance at the permissible error of oscillation frequency in the limits of $1 \cdots 2 \%$, that is, in the limits of $500 \mathrm{pF}$. When reducing oscillation amplitude to the level of $5 \mathrm{~V}$ as well, one could speak about maximum oscillation frequency only about $200 \mathrm{kHz}$. Thus, it is not possible to realize maximum frequency properties of the amplifier in the limits of OS with CFOA with current output under output oscillation

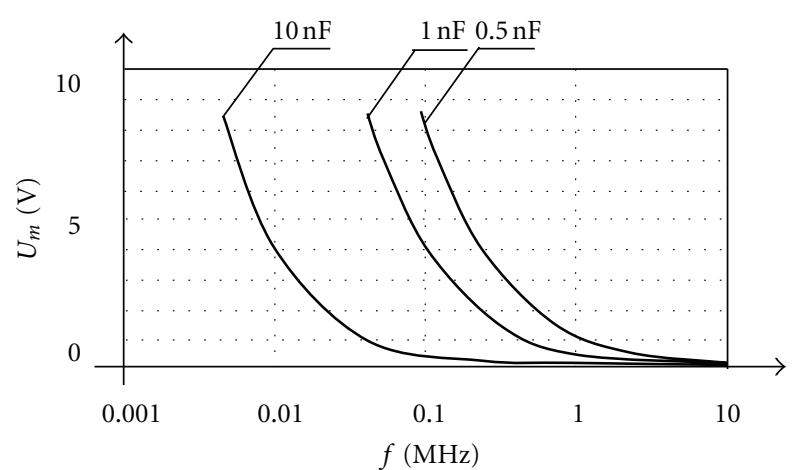

FIGURE 6: Dependence of maximum amplitude of the integrator output voltage on signal frequency.

TABLE 2: Comparison of circuits with two integrators.

\begin{tabular}{lcc}
\hline Parameters & Figure 5(a) & Figure 5(b) \\
\hline $\begin{array}{l}\text { Number of amplifiers } \\
\begin{array}{l}\text { Number of passive } \\
\text { elements }\end{array}\end{array}$ & 3 & 2 \\
Outputs & 6 & 5 \\
$\begin{array}{l}\text { Harmonic rate, dB } \\
\text { Frequency range in OS } \\
\text { with CFOA, MHz }\end{array}$ & $-(85 \cdots 90)$ & $-(58 \cdots 80)$ \\
\hline
\end{tabular}

amplitude of some $5 \mathrm{~V}$. Perhaps for this reason, the oscillation amplitude at frequencies above $1 \mathrm{MHz}$ in the works $[10,11]$ is less than $1 \mathrm{~V} \mathrm{p}$-p.

Figure 6 shows the dependences in diagrams. Using the diagrams and knowing the value of the integrator capacitor capacitance one can easily estimate maximum value of output voltage amplitude.

Thus, the comparison of basic OS parameters with two integrators is tabulated in Table 2.

The main advantage of OS circuits with CFOA mentioned by the authors of $[3,4,6]$ is the possibility to connect RC-elements of frequency and oscillation amplitude control by the common wire. It is certainly an important advantage, but it is not crucial today, as the time of bulky mechanical doubled capacitors and potentiometers for frequency changing has passed. Today frequency is controlled by D/A converters and capacitors with electronic changing, and the problem is not so pressing.

\section{Conclusion}

The use of current feedback operational amplifiers (CFOAs) in oscillatory systems of generators instead of potential feedback amplifiers leads to

(i) the increase of harmonic distortions,

(ii) the reduction of generated frequency range under equal oscillation amplitude. 
Thus, oscillatory system of electric signals generator with CFOA does not have considerable advantages and in respect of harmonic level and frequency properties even yields to known oscillatory systems with traditional amplifiers.

\section{References}

[1] Analog Devices: $60 \mathrm{MHz}, 2000 \mathrm{~V} / \mu \mathrm{s}$ Monolitic Op Amp AD844, 2012, http://www.analog.com/static/imported-files/ data_sheets/AD844.pdf.

[2] Burr-Brown: Wide-Bandwidth Operational Amplifier OPA622, 2012, http://focus.ti.com/lit/ds/symlink/opa622.pdf.

[3] Yu. K. Rybin, Electronic Devices for Analog Signal Processing, Springer, Dordrecht, The Netherlands, 2011.

[4] M. T. Abuelma' atti, "Identification of a class of two CFOAbased sinusoidal RC oscillators," Analog Integrated Circuits and Signal Processing, vol. 65, no. 3, pp. 419-428, 2010.

[5] M. T. Abuelma'atti, "New two CFOA-based sinusoidal RC oscillators with buffered outlet," Analog Integrated Circuits and Signal Processing, vol. 66, no. 3, pp. 475-482, 2011.

[6] M. T. Abuelma'atti and S. M. Al-Shahrani, "Novel CFOAbased sinusoidal oscillators," International Journal of Electronics, vol. 85, no. 4, pp. 437-441, 1998.

[7] A. M. Soliman, "Applications of the current feedback operational amplifiers," Analog Integrated Circuits and Signal Processing, vol. 11, no. 3, pp. 265-302, 1996.

[8] A. M. Soliman, "Current feedback operational amplifier based oscillators," Analog Integrated Circuits and Signal Processing, vol. 23, no. 1, pp. 45-55, 2000.

[9] P. A. Martínez, S. Celma, and I. Gutiérrez, "Wien-type oscillators using $\mathrm{CCII}^{+}$," Analog Integrated Circuits and Signal Processing, vol. 7, no. 2, pp. 139-147, 1995.

[10] A. M. Soliman, "Generation of current conveyor based oscillators using nodal admittance matrix expansion," Analog Integrated Circuits and Signal Processing, vol. 65, no. 1, pp. 4359, 2010.

[11] D. K. Srivastava and V. K. Singh, "Single-capacitor-controlled oscillators using a single CFOA," in Proceedings of the International Conference on Circuits, System and Simulation (IPCSIT '11), vol. 7, IACSIT Press, 2011. 

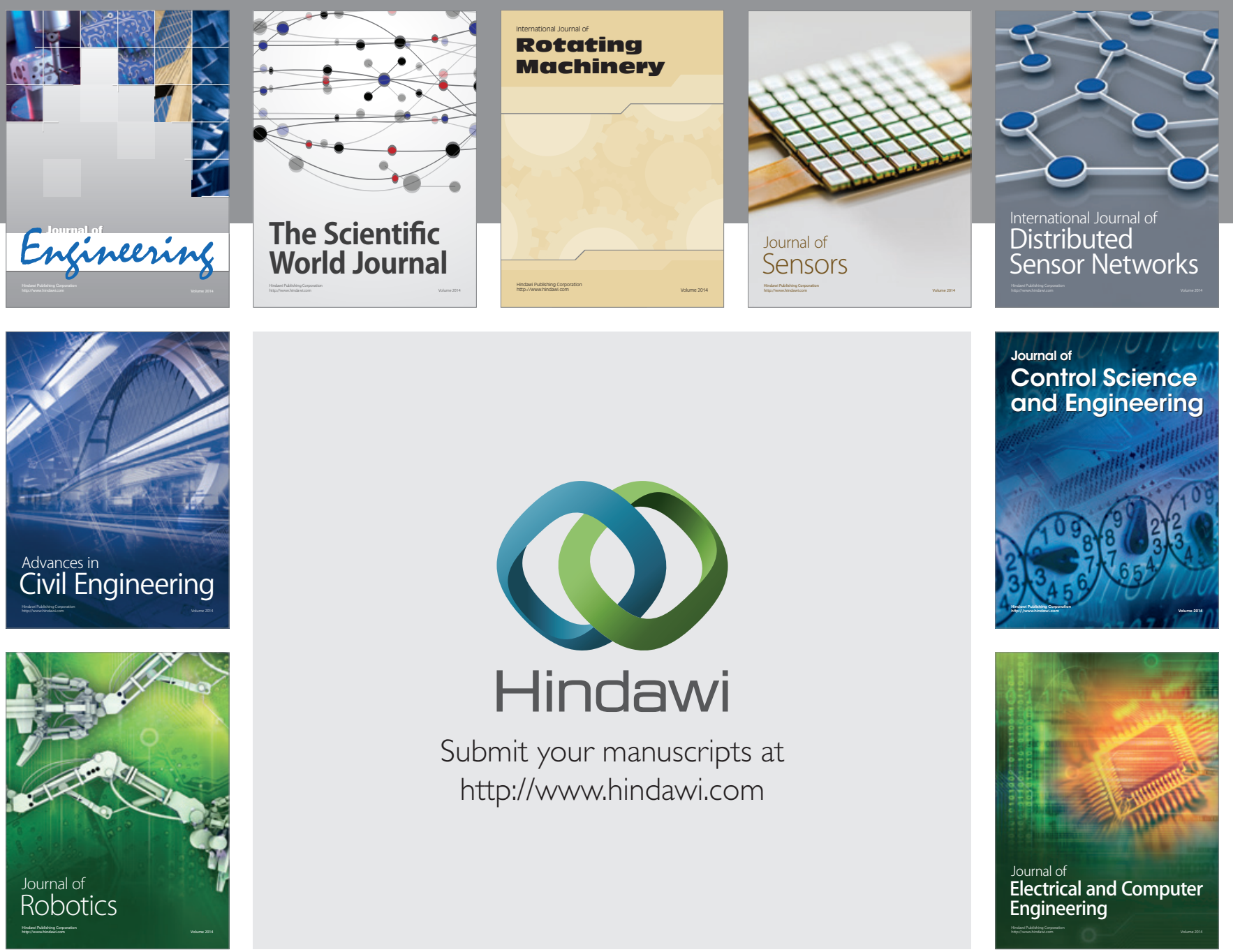

Submit your manuscripts at

http://www.hindawi.com
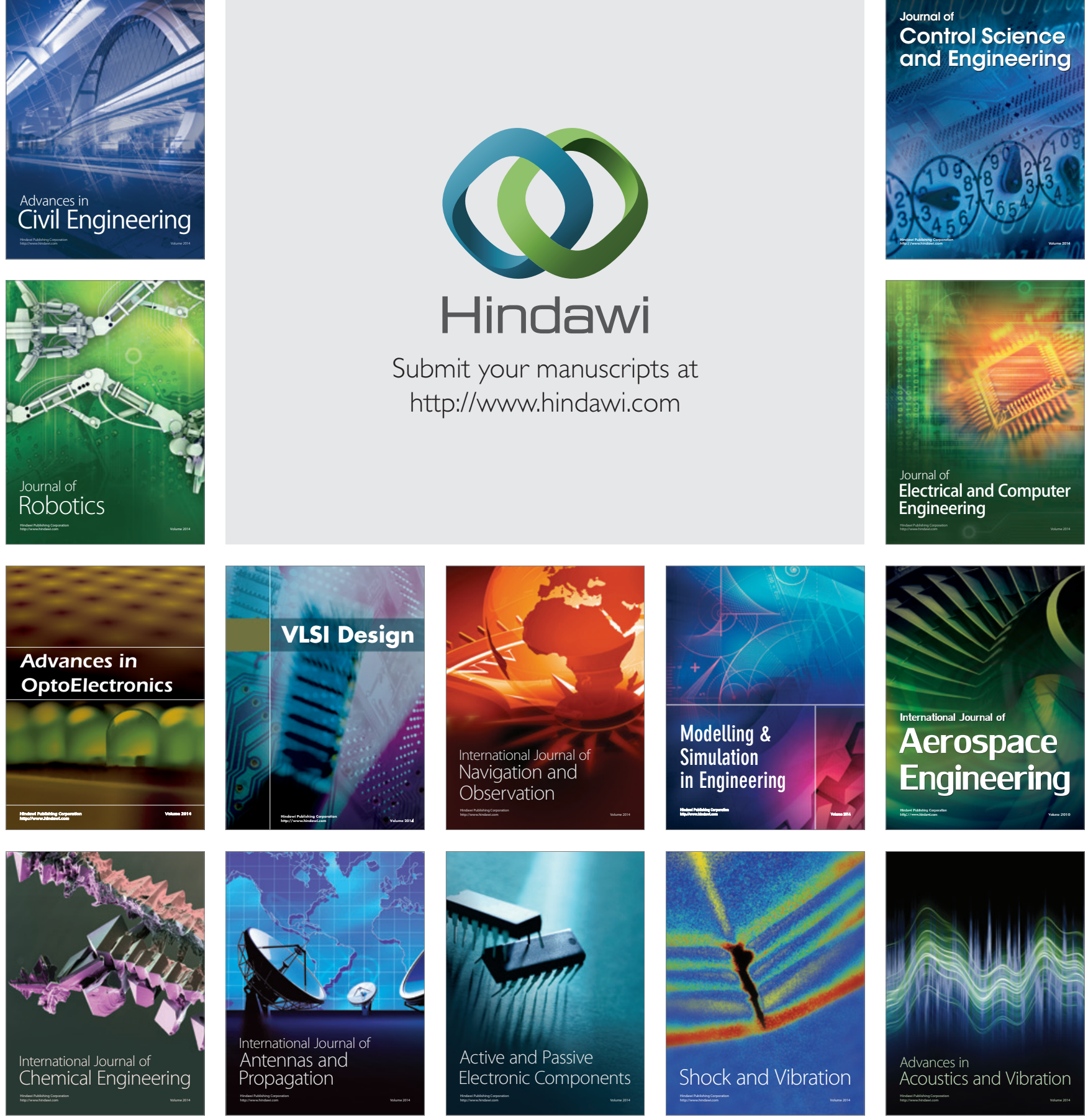